\title{
Le ricette della Lozana: cuoca, medichessa, estetista e fattucchiera
}

\author{
Lozana's recipes: cook, medician, beautician and witch
}

Carla Perugini

carla49@gmail.com

Università degli Studi di Salerno

\begin{abstract}
Riassunto: In un breve giro d'anni, fra Quattro e Cinquecento, in Italia come in Spagna, si moltiplicarono i libri di cucina, codificando un canone che rimarrà tale fino ai nostri giorni, come pure gli Antidotari, con i rimedi scientifici o presunti tali per le diverse malattie, in primis il mal francese, e ancora i libri dei segreti con consigli su ogni problema domestico e sulle cure cosmetiche, nonché i prontuari a disposizione di chi volesse usare di malefici e pronostici.

Anche ne La Lozana andaluza si sprecano ricette e consigli d'ogni genere, e tuttavia l'ironia e la vis comica del suo autore travolgono quanto di prescrittivo e normativo caratterizza il genere, sovvertendo sia la lingua sia i contenuti adottati dall'autore.
\end{abstract}

Parole chiave: Lozana andaluza, cucina, estetica, medicina, magia.

Abstract: In a short span of years, between the fifteenth and sixteenth centuries, in Italy as in Spain, cookbooks multiplied, codifying a canon that will remain so to our days, as well as the Antidotaries, with scientific or presumed remedies for the various diseases, primarily the French pox, and the books of secrets with advices on every domestic problem and on cosmetic treatments, as well as the handbooks available to those who wanted to use spells and predictions.

Even in La Lozana andaluza recipes and advices of all kinds are wasted, but its author's irony and vis comica overwhelm the prescriptive and normative character of the genre, subverting both the language and the contents adopted by Francisco Delicado.

Keywords: Lozana andaluza, cooking, cosmetics, medicine, magic. 
Prima viene lo stomaco, poi viene la morale.

Bertold Brecht

\begin{abstract}
Yo sé ensalmar y encomendar y santiguar cuando alguno está aojado, que una vieja me vezó, que era saludadera y buena como yo. Sé quitar ahitos, sé para lombrices, sé encantar la terciana, sé remedio para la cuartana y para el mal de la madre. Sé cortar frenillos de bobos y no bobos, sé hacer que no duelan los ríñones y sanar las renes, y sé medicar la natura de la mujer y la del hombre; sé sanar la sordera y sé envolver sueños; sé conocer en la frente la fisionomía y la quiromancía en la mano y prenosticar (Delicado 2004: XLI, 233).
\end{abstract}

Con questo abile richiamo alle proprie capacità, costruito a mo’ di moderno annuncio pubblicitario ma seguendo i tradizionali strumenti retorici del parallelismo, delle rime e delle assonanze interne, dell'anafora e del chiasmo, la protagonista dello straordinario Retrato de la Lozana andaluza presentava enfaticamente sé stessa al proprio autore e complice Francisco Delicado, in quella originalissima coabitazione di scrittore e personaggio che con ogni probabilità era effettivamente esistita nella Roma licenziosa e permissiva in cui i due si trovarono a vivere, e che poi fu ritratta nella finzione e metafinzione del libro, che li vede conversare, mangiare assieme, scambiarsi omaggi e prestazioni, nonché informazioni sulla cura del male che li affliggeva entrambi.

Con la stessa sicumera e ostentazione delle proprie virtù taumaturgiche, qualche decennio più tardi il medico fisico, chirurgo, inventore ed alchimista Leonardo Fioravanti avrebbe magnificato i suoi ingegnosi ritrovati in svariatissimi ambiti: dai miracolistici balsami e panacee universali agli invincibili strumenti di offesa e di difesa nell'arte della guerra, dall'invenzione di vascelli inaffondabili ai petulanti consigli di politica economica suggerita con nonchalance a duchi e cardinali. ${ }^{1}$ Entrambi, la meretrice andalusa (ovvero il suo scrittore) e il medico bolognese, ci appaiono esemplari portavoce di quella moderna curiositas che nel Rinascimento spinse gli spiriti più audaci, senza distinzioni di diplomi o di etichette, a cimentarsi negli ambiti più vari del sapere, dallo scoprire terre incognite e misteri del cosmo al rinnovare modelli invecchiati nelle arti o a escogitare rimedi per epidemie sconosciute, come per quel male battezzato francese, napoletano o con mille altri nomi su cui s'impose infine sifilide, e che imperversò in Europa per tutto il secolo XVI.

Tornando all'arringa iniziale di Lozana, essa è paradigmatica delle strategie discorsive che sostengono il libro, il quale riflette nella sua struttura formale la medesima libertà di pensiero e di azione dei due principali attanti: la prostituta e l'autore. Come né l'una né l'altro, infatti, si preoccupano di aderire a rigide norme di comportamento, aggirando, senza scrupoli, tradizionali divisioni di classe, sesso e credo religiosi, così Delicado provvede a far saltare egualmente ogni canone letterario e linguistico. La Lozana andaluza è un libro inclassificabile, privo di centro e pieno di periferie, in cui ogni episodio, ogni aneddoto, ogni persona che si trovino a portata di sguardo e di quaderno del chierico andaluso vengono fulmineamente catturati e annotati («no quiero ir porque dicen después

1 A Fioravanti sono state dedicate due bellissime biografie: Camporesi 2021 e Eamon 2019.

SCRIPTA, Revista internacional de literatura i cultura medieval i moderna, núm. 18 / desembre 2021 / pp. 223-232 ISSN: 2340-4841 · doi:10.7203/SCRIPTA.18.22777 
que no hago sino mirar y notar lo que pasa para [e]screbir después, y que saco dechados», Delicado 2004: XVII, 100). Fosse pure per una sola battuta, ogni personaggio, anche quello che magari non comparirà mai più fra le pagine, viene individuato alla stregua dei protagonisti con un nome, $\mathrm{o}$ anche più di uno, vista la predilezione dello scrittore per le endiadi.

Racconti folklorici, cronaca cittadina, leggende agiografiche, battute equivoche, incontri erotici, considerazioni parafilosofiche...: ogni aspetto di Roma e dei suoi abitanti viene catturato in presa diretta e comunicato a chi legge o ascolta il libro, ieri come oggi. Questa modalità di scrittura, se da un lato le conferisce vivacità, ritmo e originalità, dall'altro sconta lo stile rapsodico con frequenti ellissi nella cronologia -che risulta del tutto arbitraria- e con numerose contraddizioni nella storia dei due amanti e compari, Lozana e Rampín, nonché negli esiti del testo, che l'autore dà più volte per concluso, mentre viene smentito dai vari finali che si succedono l'un all'altro, fra velleità moralistiche, parodie di discussioni dotte e inevitabile ricorso ai doppi sensi anche nella narrazione di un dramma quale fu il Sacco di Roma del 1527. Detto in altri termini: il libro è, dal principio alla fine, un'apologia di eros, privo di zavorre ideologiche o religiose, un eros raccontato e rappresentato senza veli e senza infingimenti, sostenuto da un linguaggio indifferentemente popolare e colto, ambiguo e diretto, da intendersi a volte alla lettera, ma molto più spesso come metafora.

Sulla cultura di Delicado, al di là del topos della modestia, comune a tutti i suoi contemporanei, e delle invocazioni a essere emendato da persone più dotte di lui ${ }^{2}$ (tradizionale luogo comune di qualsiasi prologo e dedica), non credo ci possano essere dubbi: ne fanno fede i frequenti rimandi a classici dell'antichità sia greca sia romana, le allusioni a opere moderne analoghe per argomento, ambientazioni e personaggi, ${ }^{3}$ o a monumenti della tradizione ispanica come El Cid o il Romancero. Mentre nella Lozana il Delicado parroco della chiesetta di Santa Maria in Posterula prudentemente occulta la sua condizione di sacerdote, la rivela invece appieno nello Spechio vulgare per li sacerdoti, ${ }^{4}$ libretto finora semisconosciuto ${ }^{5}$ dedicato ai chierici stranieri che si recavano a Roma in occasione dell'anno santo del 1525. Qui il buontempone che si accompagnava a prostitute e gaudenti svela, con ortodossia dottrinale e profonda conoscenza delle sacre scritture, il suo côté osservante e devoto, che, insieme a quello godereccio e brillante, cercava, nei piaceri del corpo come in quelli dello spirito, una fuga dai tormenti che all'uno e all'altro inflisse il mal francese per ventitré anni.

$2 \mathrm{Ma}$ emendar, come dalle note al testo della mia edizione, deve intendersi come eyacular.

3 In primis La Celestina, ma numerose sono le concomitanze con la Carajicomedia, la Thebaida, con le commedie di Torres Naharro, i versi di Cristóbal de Castillejo, o con le opere licenziose di italiani quali Aretino, Berni, Divizi da Bibbiena. Riferimenti a Navagero e a Gonzalo Fernández de Oviedo, così come a Fernán Pérez Guzmán e a Nebrija, si ritrovano anche nelle sue opere successive.

4Ne ho curato recentemente l'edizione nella sezione Textos della rivista Lemir. Revista de Literatura Española Medieval y del Renacimiento (Delicado 2021).

5Lo aveva illustrato Francesco Ugolini, senza rivelarne l’ubicazione (Ugolini 1974-1975).

SCRIPTA, Revista internacional de literatura i cultura medieval i moderna, núm. 18 / desembre 2021 / pp. 223-232 ISSN: 2340-4841 ·doi:10.7203/SCRIPTA.18.22777 
Fra gli edonistici rimedi a tanta sofferenza, e considerando che gli abituali strumenti corporali per procurarsi il piacere sessuale erano ormai inservibili in quanto piagati e doloranti, oltre che contagiosi (ma di questo uomini e donne nel libro non sembrano fare gran caso), spicca il ruolo assegnato alla cucina, che nel Retrato è continuamente evocata, ora sotto forma di ricette, ora di pranzi e cene organizzati con le prostitute e i loro clienti. In particolare, le comidas a cui si viene invitati a casa della Lozana non sono mai veramente offerte agli ospiti, visto che l'astuta andalusa fa sì che siano loro a pagarne le spese, grazie alla sua eloquenza irresistibile, a cui dà una mano anche il fido Rampín, che sa come non restituire il resto del denaro affidatogli o come comprare sempre il meglio sul mercato del cibo e del vino. Cibo e vino che fin dall'antichità entravano non solo nelle ricette culinarie ma anche in quelle mediche. Rolf Eberenz, ricordando che non mancavano mai nei ricettari sezioni riservate alle preparazioni per malati e convalescenti, afferma:

Entre la medicina y la alimentación siempre han existido relaciones estrechas y complejas. Así, la mayoría de las obras médicas contienen referencias más o menos detalladas a la comida y a las bebidas que se estiman más adecuadas en cada situación. Estos consejos son particularmente frecuentes en los tratados de prevención y curación de la peste (Eberenz 2018: 1076).

Così pure Michelangelo Zaccarello sostiene che «l'intervento sulla nutrizione era di fatto l'unica terapia farmacologica disponibile» (Zaccarello 2012: 9). Seguendo la teoria umorale che da Ippocrate e Galeno, attraverso le scuole mediche arabe, ebraiche e salernitana, era giunta, quasi mai contestata, fino al Rinascimento, si riteneva che gli alimenti dovessero equilibrare gli umori in eccesso o in difetto nell'organismo. La presenza ineludibile della teoria fece sì che ai fisici non si richiedesse un'autentica competenza dell'anatomia e delle malattie del corpo umano, bensì lo studio e la conoscenza dei sacri testi della medicina, sui quali si svolgeva l'esame per ottenere la laurea. L'esperienza poi faceva il resto, ma a questo punto erano molto più titolati gli speziali, che nelle loro botteghe avevano a disposizione per ogni esigenza numerosi antidotari su farmacopea e terapie, ${ }^{6}$ o anche i praticoni e le curanderas, che contendevano con successo i pazienti ai medici laureati, specie se di mali femminili si trattava. In molti trattati medici riscontriamo la sdegnosa ripulsa nei confronti di quelle mulierculae, donnicciole, «povere femminelle» (Camporesi 2021: 13), ${ }^{7}$ che, spesso analfabete e sempre dietro modeste ricompense, applicavano i segreti trasmessi da madre in figlia, dimostrando che, come sosteneva Leonardo Fioravanti nel suo libro Della fisica (1582), «più vale un palmo di buona e vera esperienza che non fa una canna di incerta teorica» (Camporesi 2021: 327). Nel suo ruolo di saludadera e curandera, anche Lozana soffia clienti ai medici ufficialmente riconosciuti, gira per le case delle cortigiane spacciando loro flaconi d'acqua fresca per esemplari unici di

6 Ricordiamo che lo stesso Delicado nel suo libello El modo de adoperare el legno de India Occidentale aveva magnificato il proprio rimedio contro il mal francese, a base di guayaco (Delicado 2004 e 2021).

7 L'espressione è tratta da un famoso libro dei segreti del reverendo donno Alessio Piemontese (in realtà Girolamo Ruscelli), del 1559, nella dedica ai lettori. 
cosmetici miracolosi e recita salmi strampalati che guarirebbero le malattie veneree. L'eredità celestinesca di scongiuri e incantesimi è però solo apparente: a differenza di altre discendenti della vecchia Celestina, la bella andalusa non crede affatto alla magia, né bianca né nera. Ciò che propina ai suoi pazienti è quello che intuisce che si aspettino, consapevole che «la melezina ha de estar en la lengua» (Delicado 2004: XXVI, 176).

Lozana è l'incarnazione di quei saperi delle donne negli ambiti della cura, della cosmetica, della cucina e dei riti delle superstizioni popolari, che si tramandavano per lo più in forma orale $\mathrm{o}$ su qualche scartafaccio ereditato in famiglia, ma che conobbero grande diffusione e successo attraverso i libri dei segreti, ricettari o prontuari fai da te per ogni necessità quotidiana, dalla colorazione dei capelli alla confezione di inchiostro artigianale, dai rimedi per non far ammuffire il vino alla tintura delle stoffe, dalle diete per gli infermi o per i bambini alle soluzioni contro invasioni casalinghe di topi o insetti vari. Appartenenti alla tipologia dei libri-biblioteca, perché composti da compilatori che attingevano, manipolandole, a fonti tanto erudite quanto popolari, scritti in parte in volgare in parte in latino, da foglietti volanti in mano a imbonitori e ciarlatani conobbero poi un'esplosione di edizioni a stampa fra Cinquecento e Settecento (Palmero 2014: 271).

Per quanto riguarda la cucina, sebbene anche nel Medioevo, «con una singolare contemporaneità in tutta Europa» (Bertolini 1998: 738), avessero circolato delle raccolte scritte di ricette, è solo col Quattrocento che esse assumono una forma paradigmatica. Ciò che trasformerà un manipolo di annotazioni alimentari in un libro di cucina sarà il paratesto (titolo, sottotitoli, prologo, iconografia...) e la destinazione a un pubblico invitato a intervenire con aggiunte, interpolazioni e soprattutto messa in pratica di quanto è presente nel libro (Peyrebonne 2009: 487). Pur essendo un testo prescrittivo (sintomatico l'uso dell'imperativo) perché destinato alle tavole dei ricchi, secondo dei rigidi cerimoniali e una netta suddivisione dei compiti fra chi aveva in carico la preparazione dei cibi e chi la loro presentazione, il libro di cucina si prestava a interventi del lettore, che poteva modificarne non solo i procedimenti e gli ingredienti ma gli stessi titoli. Prova ne siano le successive edizioni del Lybre de doctrina Pera ben Servir: de Tallar: y del Art de Coch del maestro Roberto di Nola che, dall'edizione catalana del 1520, diventa Libro de cocina compuesto por maestre Ruberto de Nola nell'edizione toledana del 1525 e quindi Libro de guisados, manjares y potajes intitulado libro de cozina nel 1529 a Logroño, spostando l'interesse dall'arte del trinciare a quella del cucinare e del governare la casa del signore.

Senza entrare nella discussione sull'esistenza o meno di una Fachsprache dei libri di cucina, «tanto a livello microtestuale (lessico e sintassi) quanto macrotestuale (organizzazione interna, partizione e ordine del testo ecc.)» (Lubello 2001: 232), ci limiteremo a rilevare le peculiarità del nostro testo di riferimento.

Concordiamo con quanto ha citato dal libro di Massimo Montanari Il cibo come cultura la filologa Anna Martellotti:

L'analogia fra cibo e linguaggio, che abbiamo messo a confronto come sistemi di segni oltre che (nel caso del cibo) di realtà materiali, li connota entrambi come codici di comunicazione, che, all'interno

SCRIPTA, Revista internacional de literatura i cultura medieval i moderna, núm. 18 / desembre 2021 / pp. 223-232 ISSN: 2340-4841 ·doi:10.7203/SCRIPTA.18.22777 
e all'esterno delle società che li esprimono, trasmettono valori simbolici e significanti di varia natura (economici, sociali, politici, religiosi, etnici, estetici ecc.). Come la lingua parlata, il sistema alimentare contiene e trasporta la cultura di chi lo pratica, è depositario delle tradizioni e dell'identità di gruppo (Montanari 2007 in Martellotti 2012: IX). ${ }^{8}$

Naturalmente non possiamo parlare della Lozana andaluza come se di un libro di cucina si trattasse, però essa è significativamente presente nel libro, non solamente a livello di citazioni di alimenti crudi o cotti secondo la gastronomia romana $\mathrm{o}$, più frequentemente, andalusa, ma con significati traslati che rimandano alla cifra erotica sottesa a tutta l'opera. Se dunque la prima impressione che si ricava dagli inesauribili elenchi di ingredienti e rielaborazioni mangiarecce è di ammirazione per le virtù culinarie della protagonista, a una lettura non ingenua essi si rivelano altrettanti segni di un universo linguistico e semantico sotto le insegne di eros, molto diffuso peraltro nella letteratura licenziosa del tempo. Segni che il ristretto novero di sodali a cui il chierico andaluso si rivolgeva a Roma per distrarsi dai patimenti del suo male, o il più vasto pubblico per il quale darà alle stampe l'edizione veneziana (1530), dovevano immediatamente cogliere e tradurre nel loro autentico significato, al di là dell'uso disinvolto degli eufemismi, delle iperboli, delle metafore, degli hapax legomenon, delle antifrasi e dei termini equivoci, equivoci che a volte includevano mamotretos interi, come nei fasulli racconti della fuitina di Aldonza (non ancora Lozana) con Diomede, degli amori contrastati del giovane Coridón e della donzella Polidora, dell'asino Robusto e del suo padrone Porfirio che lo volle laureato o ancora del presunto esilio finale nell'isola di Lipari da parte di Lozana e Rampín.

Non a caso la prima xilografia che compare a lato della pseudobiografia infantile dell'eroina è una granada, ben aperta da un taglio che mette in mostra i suoi numerosi e rossi chicchi, auspicio tradizionale di fecondità e succosi sapori. Il rimando al sesso femminile è sottolineato dall'analogo toponimo, in un contesto di termini legati a quel prolifico lignaggio di zie, nonne, padri e madri, che stanno per organi genitali e relativi sfruttatori. Mostrando una precocità foriera di un futuro colmo d'incontri ravvicinati, Lozana si vanta di aver cucinato patriotticamente per il padre e per tutto il parentado, mentre da adulta si trova a dover accontentare gli stranieri. Invero la cosa non doveva dispiacerle più di tanto se, come lei stessa confessa: «desde chiquita me comía lo mío, y en ver hombre me desperezaba, y me quisiera ir con alguno» (Delicado 2004: VII, 40). Reciprocamente, nella Celestina, nel descrivere «las pequeñas tetas» di Melibea, Calisto ammette che «se despereza el hombre cuando las mira» (Rojas 2000: I, 45).

Il secondo mamotreto è un vero inno alla cucina andalusa, nelle sue varianti semitiche, in particolare arabe, secondo il giudizio, a volte estremizzante, di Federico Corriente (Corriente 2010 e 2017). Ci sembra di cogliere, in queste pagine dedicate da Delicado ai piatti della sua giovinezza, la gioia della moltiplicazione, dell'eccesso, dell'enfasi iperbolica, che ben si adattano all'insaziabile voracità della sua connazionale, le cui due bocche chiedono sempre nuovi pasti (Hernández Cobo 2015):?

8 Pioniere dell'affermazione dell'omologia tra cibo e linguaggio fu Claude Lévi-Strauss con il suo articolo «Le triangle culinaire» (Lévi-Strauss 1965).

9 Durante una partita a carte fra lei, Rampín e due sfaccendati, la posta che propone è trasparente: «Lozana: Yo porné

SCRIPTA, Revista internacional de literatura i cultura medieval i moderna, núm. 18 / desembre 2021 / pp. 223-232 ISSN: 2340-4841 ·doi:10.7203/SCRIPTA.18.22777 
Sabía hacer hojuelas, prestiños, rosquillas de alfajor, textones de cañamones y de ajonjolí, nuégados, jopaipas, hojaldres, hormigos torcidos con aceite, talvinas, zahinas y nabos sin tocino y con comino, col murciana con alcaravea. Y olla reposada no la comía tal ninguna barba. Pues ¿boronía no sabía hacer? ¡Por maravilla! Y cazuela de berenjenas, mojíes en perfición, cazuela con su ajico y cominico y saborcico de vinagre: ésta hacía yo sin que me la vezasen. Rellenos, cuajarejos de cabritos, pepitorias y cabrito apedreado con limón ceutí. Y cazuelas de pescado cecial con oruga y cazuelas moriscas por maravilla y de otros pescados que sería luengo de contar. Letuarios de arrope para en casa, y con miel para presentar, como eran de membrillo, de cantueso, de uvas, de berenjenas, de nueces. Y de la flor del nogal para tiempo de peste, de orégano y hierbabuena para quien pierde el apetito. Pues ¿ollas en tiempo de ayuno? Estas y las otras ponía yo tanta hemencia en ellas que sobrepujaba a Platina De voluptatibus y Apicio romano De re coquinaria (Delicado 2004: II, 19-20).

In questo delirio verbale ci si perde dietro tanti odori e sapori. Vorrei soffermarmi solo sull'allusione ai giorni «de ayuno», tanti e frequenti, che la Chiesa imponeva nel corso dell'anno. Certo è che fra gli ecclesiastici e i patrizi i banchetti cosiddetti di magro evitavano solo i cibi proibiti, in primis la carne, ma non l'abbondanza e la ricercatezza di decine d'altri piatti, come ben illustrano i libri di cucina di cuochi famosi che officiavano alle loro mense, quali Bartolomeo Scappi, Giovanne [sic] de Rosselli, Eustachio Celebrino, Cristoforo di Messisbugo, Domenico Romoli detto il Panonto, Michele Savonarola o Maestro Martino, dei quali Claudio Benporat ha riportato sconcertanti documenti di convivi dalle interminabili portate (Benporat 2007).

Una spia della poca diligenza dei fedeli a seguire le prescrizioni di mortificazione della carne sta in quella «tanta hemencia» che nel brano su citato rivendica Lozana, ovvero «vehemencia, eficacia» in posizione anticipatrice dei due volumi più ricercati dagli umanisti italiani (dobbiamo contare fra di loro anche una prostituta sine litteris?). Solo libro di cucina dell'antichità romana giunto fino a noi, il De re coquinaria del controverso Apicio fu trascritto in vari monasteri durante il Medioevo, subendo parecchie manipolazioni. Furono gli umanisti italiani, in particolare Enoch d'Ascoli, a riportarlo in Italia a metà del Quattrocento, e a lanciarlo come «une véritable mode» (Laurioux 1994: 33), e ad esso s'ispirò Bartolomeo Sacchi, detto il Platina, per il suo famoso trattato De honesta voluptate et valetudine (1475). È piuttosto curioso che Delicado metta in bocca alla sua smodata eroina un titolo come quello del Platina, un teorico, per dirla con Camporesi «della cucina "regolata" secondo il gusto della misura $[. .$.$] moralisticamente orientato verso la$ conservazione della "buona salute"»(Camporesi 2018: 66). Ma a contraddizioni e smentite ci hanno abituato le sue pagine.

Nonostante l'ammirata grassezza di Lozana testimoni, con sfacciato orgoglio, l'affratellamento, alla sua tavola, di pietanze romanesche e spagnole, giudaiche e cristiane, la sua predilezione va, e non poteva essere diversamente, a quelle della tradizione andalusa. Si vanta di saper preparare pimentadas e capirotadas, cazuelas mojies e cabrieto apedreado, salmorejo e lebrada e mille altre specialità con cui attirarsi protezioni e clienti. Inoltre è un'intenditrice di vini, fra cui predilige quelli di Martos, non a caso patria del suo autore. Patria la cui lingua Delicado esalta al di sopra di tutte le altre presenti nella penisola

mi papo. Valerio: ¿Cuál, señora? Lozana: Todos dos, que hambre tengo» (Delicado 2004: 175).

SCRIPTA, Revista internacional de literatura i cultura medieval i moderna, núm. 18 / desembre 2021 / pp. 223-232 ISSN: 2340-4841 ·doi:10.7203/SCRIPTA.18.22777 
iberica, come dirà esplicitamente nella introduzione al terzo libro del Primaleón, nell'edizione che ne curò nel 1534 a Venezia per Nicolini de Sabbio e Giovan Battista Pedrezano. ${ }^{10}$

A suo dire in Andalusia, che egli chiama anche Castilla la baja, perché ripopolata da castigliani dopo la reconquista, si parla una lingua equiparabile a quella toledana, mentre nel resto della penisola sono tutte barbare: «Mas ni los gallegos ni vizcaínos ni navarros ni aragoneses ni portogueses ni catalanes no conforman sus bárbaras lenguas con los castellanos como lo hacen los de Castilla la baja que son de Toledo acá yuso» (Delicado 2004: 449). Gli andalusismi della sua Lozana (la cui paternità svela proprio in quest'Introduzione) sono di tipo evocativo, attraverso il ricordo di personaggi della storia o della cultura regionali o anche delle scoperte archeologiche antiche e recenti, piuttosto che linguistici, e più lessicali che fonetici. Possiamo ricordare l'accentuazione dell'imperfetto (tiñié per tenía), dove incontriamo anche la palatalizzazione, che si dà pure in maliñas per malignas, in engeñé per ingenié, o nel gruppo li come in callente per caliente, escallentaréis per escalentaréis, o l'epentesi della $-\mathrm{n}$ in muncho/a, lo zero fonetico al posto della $-\mathrm{r}$ finale di parola (conservá per conservar, cená per cenar), la semplificazione consonantica di ilesia per iglesia, nonché la predilezione per il diminutivo in -ico, che nel resto di Spagna nel Cinquecento aveva perso piede a favore di -illo.

Tuttavia le tesi di Delicado non vanno considerate come mere rivendicazioni nazionalistiche, se ricordiamo quanto scriveva Beinhauer:

Aunque fonéticamente diferente del castellano, más que por ejemplo el aragonés, el asturiano, el leonés y otras variedades dialectales, sintáctica y estilísticamente el andaluz, según Meyer-Lübke [...], es como una continuación del castellano, ostentando tendencias evolutivas idénticas a él (Beinhauer 1960: 225).

D'altronde lo scrittore, come portatore di una lingua pura, si rivelò un appassionato correttore dei libri di cavalleria, deturpati a suo dire dalle edizioni fattene da spagnoli ignoranti o da italiani. Per quest'ultimi arrivò a redigere un minitrattato di norme fonetiche e di pronuncia dello spagnolo al termine della sua edizione del Primaleón, trattatello di cui peraltro si appropriò, senza citarlo, il traduttore della Celestina Alfonso de Ulloa nella sua Introdutione che mostra il signor Alfonso di Uglioa a proferire la lingua Castigliana (1553).

La posizione privilegiata che detiene la cucina nel Retrato de la Lozana andaluza è dovuta a molteplici fattori, quali la prevalenza dell'esperienza sensoriale e del piacere nella protagonista del libro e nel suo autore, nella facile adattabilità al doppio senso erotico a cui si presta tutto ciò che è alimentare, ma anche a una forte componente nostalgica della patria abbandonata da troppi anni dal chierico andaluso.

La sua dichiarazione di poetica non consente equivoci:

10 La riproduco nella mia edizione de La Lozana, pp. 433-453.

SCRIPTA, Revista internacional de literatura i cultura medieval i moderna, núm. 18 / desembre 2021 / pp. 223-232 ISSN: 2340-4841 · doi:10.7203/SCRIPTA.18.22777 
y si quisieren reprehender que por qué no van munchas palabras en perfeta lengua castellana, digo que, siendo andaluz y no letrado y escriviendo para darme solacio y pasar mi fortuna que en este tiempo el Señor me había dado, conformaba mi hablar al sonido de mis orejas, que es la lengua materna y su común hablar entre mujeres (Delicado 2004: 350).

Compuse La Lozana en el común hablar de la polida Andalucía, mas hícelo por mejor la arrendar en la manera de su hablar (Delicado 2004: 448).

Affettuosa rievocazione da parte dell'uomo, ormai adulto e sperimentato, dei luoghi della propria infanzia, e di quella lingua materna che è, letteralmente, la lingua della madre, lingua femminile, parlata dalle donne, veicolo della comunicazione primaria di chi impara a esprimersi, imprinting insostituibile e incancellabile, al di là di quanto nel corso della vita si potrà acquistare o perdere.

\section{Bibliografia}

Benporat, C. (2007) Cucina e convivialità italiana del Cinquecento, Firenze, Olschki.

Beinhauer, W. (1960) «Algunos rasgos evolutivos del andaluz y el lenguaje vulgar», in Studia Philologica. Homenaje ofrecido a Dámaso Alonso, Madrid, Gredos, pp. 225-236.

Bertolini, L. (1998) «Fra pratica e scrittura: la cucina nell'Europa del tardo Medioevo», Archivio Storico Italiano, ottobre-dicembre, vol. 156, 4 (578), pp. 737-743.

Camporesi, P. (2018 [Ia ed. 1978, Bologna, Il Mulino]) Il paese della fame, Milano, Garzanti.

- (2021 [1 a ed. 1997, Milano, Il Saggiatore]) Camminare il mondo. Vita e avventure di Leonardo Fioravanti, medico del Cinquecento, Milano, Il Saggiatore.

Corriente Córdoba, F. (2010), «Los arabismos de La Lozana andaluza», Estudis Romànics [Institut d'Estudis Catalans], 32, pp. 51-72.

- (2017) «La cocina de La lozana andaluza: testimonios y ecos en las dos orillas septentrional y meridional del Mediterráneo», in Laplana Gil, J. E, Ezama Gil, M. de los A, Marín Pina, M. C., Pellicer R., Pérez Lasheras, A., Sánchez Laílla, L. (coords.), «La razón es Aurora». Estudios en homenaje a la profesora Aurora Egido, Zaragoza, Institución Fernando el Católico, pp. 545-552.

Delicado, F. (2004) La Lozana andaluza, edición de C. Perugini, Sevilla, Fundación José Manuel Lara, Clásicos Andaluces.

- (2021), «Spechio vulgare per li sacerdoti, Introducción, edición y notas de Carla Perugini, con nuevos datos bio-bibliográficos sobre El modo de adoperare el legno de India Occidentale», Lemir. Revista de Literatura Española Medievaly del Renacimiento, 25, Textos, pp. 141-188. 
Eamon, W. (2019 [1a ed. 2010, The professor of secrets. Mystery, medicine and alchemy in Renaissance Italy, Washington, DC, National Geographic Society]) Il professore di segreti. Mistero, medicina e alchimia nell'Italia del Rinascimento, Roma, Carocci.

Eberenz, R. (2018) «Discurso y léxico de la alimentación en los tratados médicos medievales y renacentistas» en Arnal Purroy, M. L., Castañer Martín, R. M., Enguita Utrilla, J. M., Lagüéns Gracia, V., Martín Zorraquino, M. A. (coords.), Actas del X Congreso Internacional de Historia de la Lengua Española, Zaragoza, Institución Fernando el Católico, II pp. 1075-1088.

Hernández Cobo, J. A. (2015) «El hambre y las ganas de entrar: doble lectura sexual en el léxico de comida y puertas en Retrato de la Lozana andaluza», Hispanófila, 175, pp. 201-212.

Lubello, S. (2001) «Il linguaggio gastronomico italiano dei secoli XIV-XVI: ultime ricognizioni», in Gualdo, R. Le parole della scienza. Scritture tecniche e scientifiche in volgare (secoli XIII-XV), Galatina, Congedo Editore, pp. 229-242.

Laurioux, B. (1994) «Cuisiner à l’Antique: Apicius au Moyen Age», Médiévales, 26, pp. 17-38.

Lévi-Strauss, C. (2004 [1a. ed. 1965, L’Arc, 26, pp. 19-29]) «Le triangle culinaire», Food \& History, 2, pp. 9-19.

Martellotti, A. (2012) Linguistica e cucina, Firenze, Olschki.

Montanari, M. (2007) Il cibo come cultura, Roma/Bari, Laterza.

Palmero, G. (2014) «La letteratura dei segreti, i "libri-biblioteca" e il vino. Un caso ligure», in Carassale A. e Lo Basso L. (a cura di), In Terra Vineata. La vite e il vino in Liguria e nelle Alpi Marittime dal Medioevo ai nostri giorni. Studi in memoria di Giovanni Rebora, Ventimiglia, Philobiblion, pp. 271-288.

Peyrebonne, N. (2009) «El paratexto de los libros de cocina en la España del siglo XVI», in Arredondo M. S., Civil P. y Moner M. (eds.), Paratextos en la literatura española (siglos XV-XVIII), Madrid, Collection de la Casa de Velázquez (111), pp. 485-496.

Rojas, F. de (2000) La Celestina. Tragicomedia de Calisto y Melibea, Barcelona, Crítica.

Ugolini, F. (1974-75) «Nuovi dati intorno alla biografia di Francesco Delicado desunti da una sua sconosciuta operetta», Annali della Facoltà di Lingue di Perugia, XII, pp. 443-616.

Zaccarello, M. (2012) «Premessa», in Treccani E. e Zaccarello M. (a cura di), Recipe... Pratiche mediche, cosmetiche e culinarie attraverso i testi (secoli XIV-XVI), Verona, Cierre Grafica, pp. 7-19. 